\title{
Studies on Fluorescence Spectral Characteristics of Lung Cancer Serum Mengrui Pan ${ }^{1, a}$, Changde Peng ${ }^{1 *, b}$, Qianying Guo ${ }^{1, c}$ \\ ${ }^{1}$ College of Physics and Electronic Engineering, Jiangsu Normal University, Xuzhou, China. \\ a1054000737@qq.com, bpengchangde@126.com, 1390090602@qq.com
}

Keywords: Lung cancer, serum, fluorescent spectrum, polarization

\begin{abstract}
The fluorescence spectral and polarized fluorescence spectral characteristics of normal and lung cancer serum solution whose concentrations are from $0.1 \%$ to $4 \%$ at the band $360 \sim 800 \mathrm{~nm}$ induced by $408 \mathrm{~nm}$ light are reported. The difference between two types of spectra has been studied. The result shows that the fluorescence intensity of lung cancer serum is stronger than the normal one but the polarization of lung cancer serum is obviously less than that of healthy person under the same concentration. The research will gives reference to the diagnosis of lung cancer.
\end{abstract}

\section{Introduction}

Recently, many techniques have been employed for the studies on the early diagnosis of cancer [1-7]. Thereinto, researches on human tissue auto-fluorescence based on fluorescent spectrum, a technique known as high sensitivity, have made a certain progress [8-10]. However, the study on the polarization of human tissues is rare. This paper provide the difference between normal human and lung cancer patients in contrast to polarized fluorescence spectra of normal and lung cancer serum.

\section{Experimental Samples and Methods}

Using the Steady State Fluorescence Spectrometer System (Type FLS900 steady-state/transient time-resolved Fluorescence Spectrometer) produced by Edinburgh Instruments Companies in UK, the real-time fluorescence measurements were collected, analyzed and exported by software.

Experimental samples. $4 \mathrm{ml}$ of intravenous fasting blood from each subject, normal human or lung cancer patient, was drawn into test tube, gently shook for even mixing of $5 \%$ heparin as anticoagulant and centrifuged at $5000 \mathrm{rpm}$ for $10 \mathrm{~min}$ one hour later [11]. Then the supernatant was absorbed into different test tube and diluted into appropriate concentration by physiological saline water (Concentration of $4 \%, 2 \%, 1 \%, 0.8 \%, 0.5 \%, 0.1 \%$ ).

Experimental methods. $2 \mathrm{ml}$ normal serum sample was put into a quartz cuvette (QC), and $2 \mathrm{ml}$ lung cancer serum sample in another QC. The fluorescence spectra of normal and lung cancer serum from 360 to $800 \mathrm{~nm}$ were scanned by $408 \mathrm{~nm}$ light with $1 \mathrm{~nm}$ scan step. A polarizer was placed between the sample and light source and an analyzer was between the sample and spectrograph. The parallel static polarized fluorescence spectra of the normal and lung cancer serum were scanned when the polarizer and analyzer were parallel and the perpendicular static polarized fluorescence were scanned when the polarizer and analyzer were crossed [12]. The whole experiments were performed at room temperature.

\section{Experimental Results and Analysis}

Comparison of fluorescence spectra between normal and lung cancer serum.The fluorescence spectra of normal serum from 450 to $600 \mathrm{~nm}$ with concentration from $0.1 \%$ to $4 \%$ are shown in Figure 1. The fluorescence spectra of lung cancer serum from 450 to $600 \mathrm{~nm}$ with concentration from $0.1 \%$ to $4 \%$ are shown in Figure 2. 


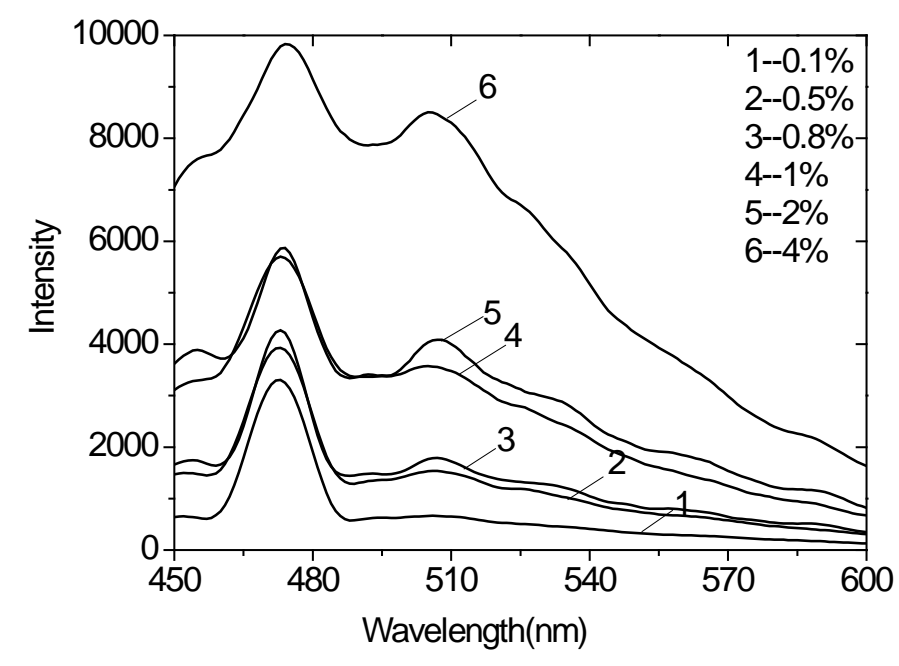

Fig 1. Fluorescence spectra of normal serum with different concentrations

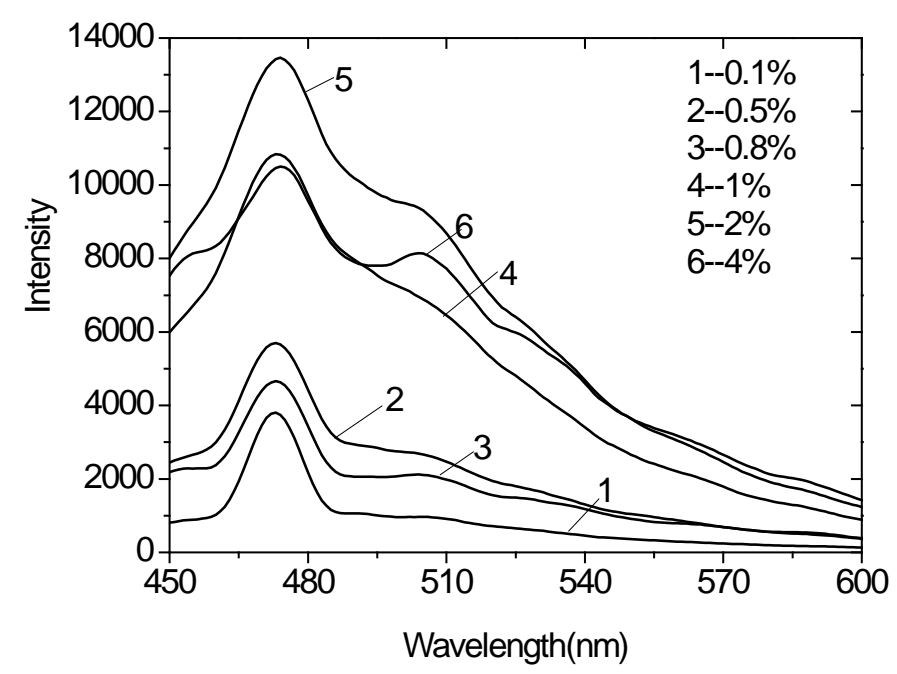

Fig 2. Fluorescence spectra of lung cancer serum with different concentrations

It is shown from Figure 1 and Figure 2 that both of the fluorescence spectra have two peaks which locate at the wavelength of $472 \mathrm{~nm}$ and $508 \mathrm{~nm}$ and one valley between the peaks. The value of peaks are increasing with the increment of serum concentration which appears more obviously near the wavelength of $472 \mathrm{~nm}$ than $508 \mathrm{~nm}$ where peak reduces gradually until disappear with the decreasing of serum concentration. Therefore, the fluorescence spectra near the wavelength of 472 $\mathrm{nm}$ are chosen to discuss.

To make the results more intuitive, the comparison of fluorescence spectra of $1 \%$ serum solution from normal human and lung cancer patients at the wavelength of $472 \mathrm{~nm}$ are shown in Figure 3. Figure 3 illustrates that the wavelength where the peak of normal fluorescence spectra located is similar to that of lung cancer, but the light intensity at the peak of lung cancer serum is close to two times of that of normal serum. Figure 4 shows the comparison of the peak intensity of fluorescence spectra between normal and lung cancer serum. 


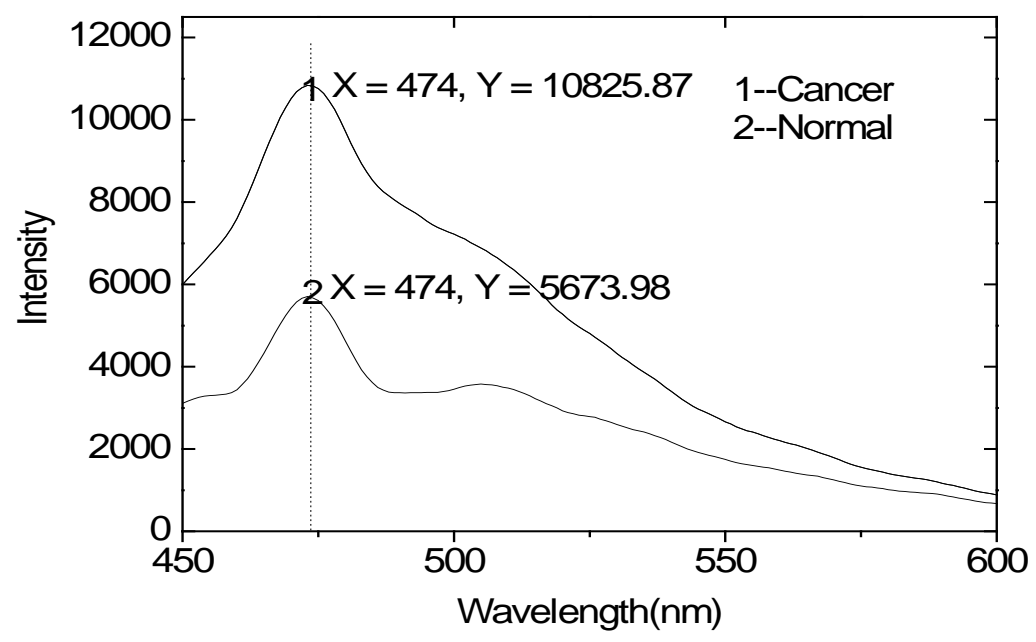

Fig 3. The comparison of fluorescence spectra of $1 \%$ normal and lung cancer serum solution

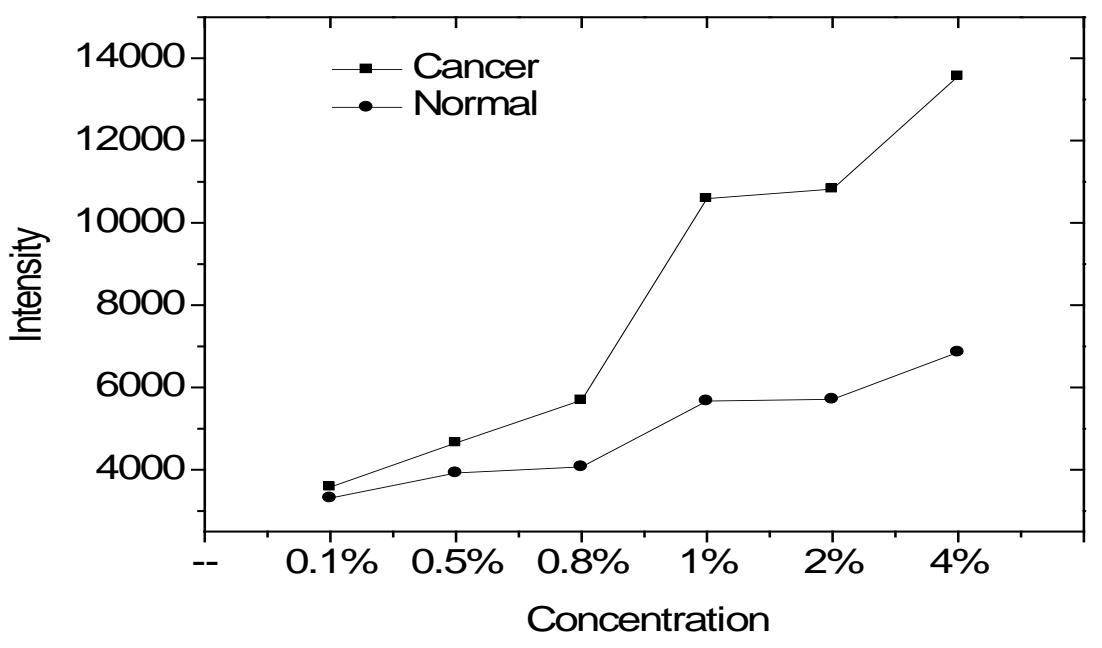

Fig 4. The comparison of the peak intensity of fluorescence spectra between normal and lung cancer serum

Figure 4 shows that the peak intensity of lung cancer serum is stronger than normal serum under the same concentration and the growth rate of the light intensity of lung cancer is obviously faster than normal serum. Therefore, the higher concentration of serum is, the greater intensity ratio of lung cancer and normal serum becomes.

Comparison of polarized fluorescence spectra between normal and lung cancer serum. Figure 5 is polarized fluorescence spectra of $0.8 \%$ normal serum after smoothing. Figure 6 is polarized fluorescence spectra of $0.8 \%$ lung cancer serum after smoothing. In Figure 5 and Figure 6 , line 1 and line 2 are respectively achieved when the polarizer and analyzer are parallel and crossed. 


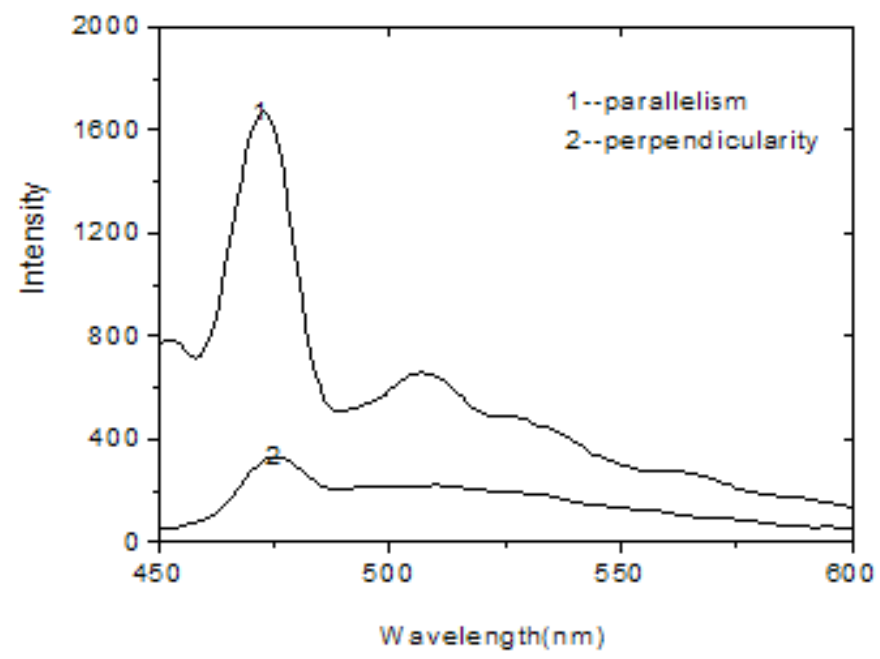

Fig 5. Polarized fluorescence spectra of $0.8 \%$ normal serum

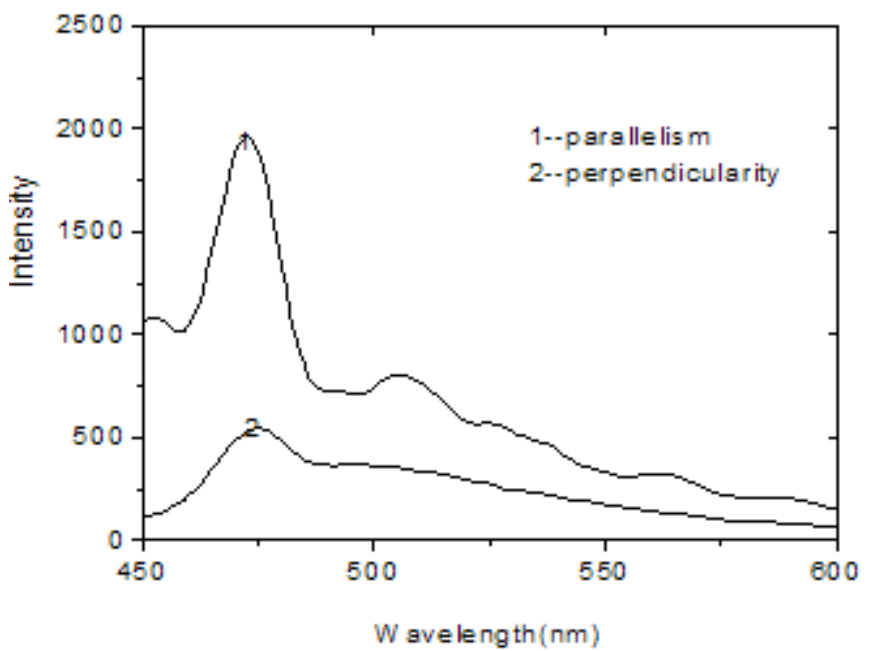

Fig 6. Polarized fluorescence spectra of $0.8 \%$ lung cancer serum

Analyzing Figure 5 and Figure 6, when two polarizers are parallel, it can be seen that the trend of polarized fluorescence spectra of normal and lung cancer serum are both similar to the unpolarized fluorescence spectra whose peaks are near the wavelength of $472 \mathrm{~nm}$ and $508 \mathrm{~nm}$. And near the wavelength of $472 \mathrm{~nm}$, the intensity of peak is relatively stronger. When the polarizer and analyzer were crossed, only the peak at the wavelength of $472 \mathrm{~nm}$ is obvious and the fluorescence intensity is obviously weaker than the parallel one. According to the fluorescence intensity, with the polarization formula

$$
\mathrm{P}=\left(\mathrm{I}_{/ /}-\mathrm{I}_{2}\right) /\left(\mathrm{I}_{/ /}+\mathrm{I}_{2}\right) \text {, }
$$

we can get that the polarization degree of normal serum is 0.6774 and that of lung cancer serum is 0.5284 . Table 1 and Table 2 show polarization degrees of normal and lung cancer serum with different concentrations. In the range of $0.1 \%$ to $4 \%$, polarization degree is increasing with the decreasing of the serum concentration. Figure 7 shows the comparison of the polarization degree of normal and lung cancer serum. It can be seen in Figure 6 and Figure 7 that the polarization degree of lung cancer serum is obviously less than normal serum under the same concentration. 


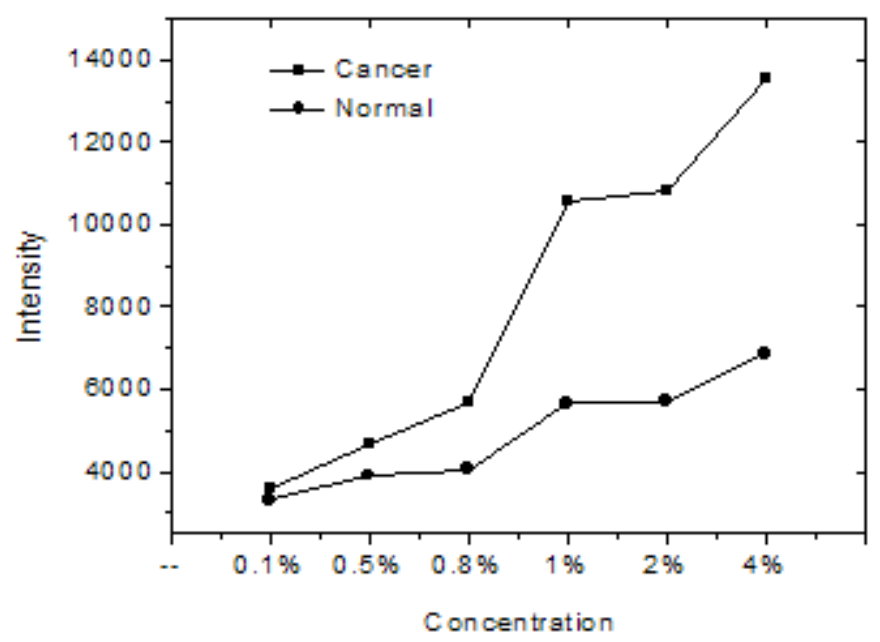

Fig 7. The comparison of polarization degree between normal and lung cancer serum

Table 1 Polarization degrees of the polarized fluorescence spectra of normal serum with different concentrations.

\begin{tabular}{lcccccc}
\hline $\begin{array}{l}\text { Serum } \\
\text { concentration }\end{array}$ & $4.0 \%$ & $2.0 \%$ & $1.0 \%$ & $0.8 \%$ & $0.5 \%$ & $0.1 \%$ \\
\hline $\begin{array}{l}\text { Polarization } \\
\text { degree }\end{array}$ & 0.4920 & 0.5297 & 0.6169 & 0.6305 & 0.6385 & 0.6774 \\
\hline
\end{tabular}

Table 2 Polarization degrees of the polarized fluorescence spectra of lung cancer serum with different concentrations

\begin{tabular}{lllllll}
\hline $\begin{array}{l}\text { Serum } \\
\text { concentration }\end{array}$ & $4.0 \%$ & $2.0 \%$ & $1.0 \%$ & $0.8 \%$ & $0.5 \%$ & $0.1 \%$ \\
\hline $\begin{array}{l}\text { Polarization } \\
\text { degree }\end{array}$ & 0.3120 & 0.3373 & 0.4303 & 0.4345 & 0.5284 & 0.5390 \\
\hline
\end{tabular}

\section{Conclusion}

In summary, this paper presented the fluorescence spectral and polarized fluorescence spectral characteristic of normal and lung cancer serum, it showed that the peak intensity of lung cancer serum fluorescence spectra is stronger than normal serum at the wavelength of $472 \mathrm{~nm}$ under the same concentration, and the growth rate of the light intensity of lung cancer serum is significantly faster than normal serum. The polarization degree of normal serum and lung cancer serum is increasing with the decreasing of the serum concentration, but the polarization degree of lung cancer serum is obviously less than the normal serum. This conclusion can provide the reference value for the early diagnosis of lung cancer.

\section{References}

[1] Liuqian Wang and Wei Ma, The progress of tumor markers for early diagnosis and prognosis evaluation in lung cancer, Journal of Modern Oncology, 2014, 22 (12).

[2] Jinlong Yang, Wenhong Peng and Kui Huang, The clinical significance of detecting serum's CEA, CYERA, NSE markers in patients with lung cancer, For all Health, 2013, 7(12).

[3] Gilad S, Lithwick-Yanai G, Barshack L, etc, Classification of the four main types of lung cancer using a micro RNA-based diagnostic assay, J Mol Diagn, 2012, 14 (5). 
[4] Yanjie Liu, Yinling Jiang, Changjiang Xu, etc, Preliminary Results of Metabolite in Serum and Urine of Lung Cancer Patients detected by Metabolomics, Chinese Journal of Lung Cancer, 2012, 15 (4).

[5] Yanli Xie, Yumei Yang, Limin Liu, etc, The clinical value of fiberoptic bronchoscopy combined with a variety of derived methods for diagnosis of lung cancer, Journal of Clinical Pulmonary Medicine, 2010, 15 (12).

[6] An Z, Chen Y, Zhang R, etc, Integrated ionization approach for RRLC-MS/MS-based metabonomics: finding potential biomarkers for lung cancer, Journal of Proteome Research, 2010, 9 (8).

[7] Zixiang Zhang, The clinical value of serum tumor markers combined detection in the diagnosis of lung cancer, The medical journal BBS, 2010, 31 (11).

[8] Xin Wang, Xiang Shen, Daping Sheng, etc, FTIR spectroscopic comparison of serum from lung cancer patients and healthy persons, Spectrochimica Acta Part A: Molecular and Biomolecular Spectroscopy, 2014, 122.

[9] Alsalhi M, Al Mehmadi A M, Abdo A A, etc, Diagnosis of liver cancer and cirrhosis by the fluorescence spectra of blood and urine, Technology in Cancer Research and Treatment, 2012, 11 (4).

[10]Xiaozhou Li, Xiangxiang Tian and Tianyue Yang, Detection of serum from lung cancer using auto-fluorescence, The Journal of Light Scattering, 2011, 23(4).

[11]Changde Peng and Jinsong Liu, Studies on Red-Shift Rules in Fluorescence Spectra of Human Blood Induced by LED, Canadian Center of Science and Education, 2013, 5 (1).

[12]Changde Peng, Ying Liu,Xiufeng Lan, etc, Research on Static Fluorescence Polarization Spectrum of Whole Blood, Acta Optica Sinica, 2006, 26 (10). 